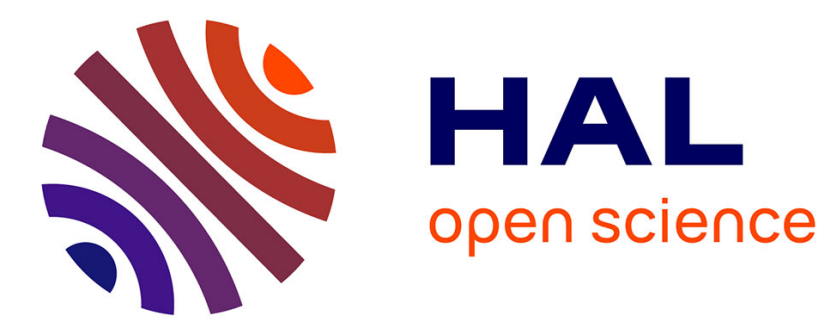

\title{
Pour une théorie évolutive des villes
}

Denise Pumain

\section{- To cite this version:}

Denise Pumain. Pour une théorie évolutive des villes. Espace Géographique, 1997, 26 (2), pp.119 134. 10.3406/spgeo.1997.1063 . hal-01760576

\section{HAL Id: hal-01760576 https://hal.science/hal-01760576}

Submitted on 6 Apr 2018

HAL is a multi-disciplinary open access archive for the deposit and dissemination of scientific research documents, whether they are published or not. The documents may come from teaching and research institutions in France or abroad, or from public or private research centers.
L'archive ouverte pluridisciplinaire HAL, est destinée au dépôt et à la diffusion de documents scientifiques de niveau recherche, publiés ou non, émanant des établissements d'enseignement et de recherche français ou étrangers, des laboratoires publics ou privés. 


\section{Pour une théorie évolutive des villes}

Mme Denise Pumain

\section{Citer ce document / Cite this document :}

Pumain Denise. Pour une théorie évolutive des villes. In: Espace géographique, tome 26, n², 1997. pp. 119-134; doi : https://doi.org/10.3406/spgeo.1997.1063

https://www.persee.fr/doc/spgeo_0046-2497_1997_num_26_2_1063

Fichier pdf généré le 02/04/2018 


\title{
Résumé
}

Les théories urbaines, en particulier économiques, reposent pour la plupart sur une conception statique de la ville (par exemple, la théorie des lieux centraux, ou la théorie des économies d'agglomération), ce qui conduit à des explications du fait urbain qui ne prennent pas en compte la genèse ni le changement, et donc réduit la pertinence des prévisions. Pour traiter des villes, qui sont des objets complexes, la théorie urbaine doit intégrer des aspects temporels, non seulement au sens de la dynamique, mais aussi du devenir, de l'histoire. Nous proposons ici les prémisses d'une théorie évolutive des systèmes de villes. Quelques éléments de cette théorie peuvent être empruntés aux modèles dynamiques d'auto-organisation inspirés des théories des systèmes physiques ou vivants, mais il faut y ajouter des propriétés, spatiales et sociales, spécifiques des systèmes urbains.

\begin{abstract}
Towards an urban evolutionary theory. - Urban theories and especially urban economic theories adopt a static conception of cities (for instance central place theory or agglomeration economies theory). As a result their explanation of the urban phenomena neglect their genesis and urban change. Therefore, possibilities of prediction are reduced. To deal with cities which are complex objects, urban theory has to integrate dynamics and history. We propose here the premisses of an urban evolutionary theory, including self-organization processes. A few elements of this theory can be borrowed from dynamic models inspired by physical or biological theories of self-organization, but they have to be complemented by some specific social and spatial properties of urban systems.
\end{abstract}




\title{
Pour une théorie évolutive des villes
}

\author{
Denise Pumain
}

Université Paris I, Institut de Géographie, 191 rue Saint-Jacques, 75005 Paris

RÉSUMÉ- - Les théories urbaines, en particulier économiques, reposent pour la plupart sur une conception statique de la ville (par exemple, la théorie des lieux centraux, ou la théorie des économies d'agglomération), ce qui conduit à des explications du fait urbain qui ne prennent pas en compte la genèse ni le changement, et donc réduit la pertinence des prévisions. Pour traiter des villes, qui sont des objets complexes, la théorie urbaine doit intégrer des aspects temporels, non seulement au sens de la dynamique, mais aussi du devenir, de l'histoire. Nous proposons ici les prémisses d'une théorie évolutive des systèmes de villes. Quelques éléments de cette théorie peuvent être empruntés aux modèles dynamiques d'auto-organisation inspirés des théories des systèmes physiques ou vivants, mais il faut y ajouter des propriétés, spatiales et sociales, spécifiques des systèmes urbains.
ABSTRACT.- Towards an urban evolutionary theory. - Urban theories and especially urban economic theories adopt a static conception of cities (for instance central place theory or agglomeration economies theory). As a result their explanation of the urban phenomena neglect their genesis and urban change. Therefore, possibilities of prediction are reduced. To deal with cities which are complex objects, urban theory has to integrate dynamics and history. We propose here the premisses of an urban evolutionary theory, including self-organization processes. A few elements of this theory can be borrowed from dynamic models inspired by physical or biological theories of self-organization, but they have to be complemented by some specific social and spatial properties of urban systems.

CITY, DYNAMIC MODEL, SELF-ORGANIZATION, URBAN SYSTEMS
Dans une analyse bibliographique récente (Pumain, Robic, 1996) on soulignait à quel point les approches théoriques des villes sont statiques. Lorsque le temps y est considéré, ce n'est le plus souvent que de manière implicite. La recherche de l'explication porte sur les structures, beaucoup plus rarement sur les modalités du changement. Aucune théorie des villes, surtout parmi celles qui sont exprimées sous forme de modèles géométriques ou mathématiques, n'a jusqu'ici convenablement intégré la dimension temporelle du phénomène urbain, le temps étant ici entendu au sens du devenir. Si opérationnelles qu'elles puissent paraître, les interprétations statiques, sur le plan explicatif, passent à côté d'une propriété essentielle de la ville, qui est son aptitude à se transformer, son caractère évolutif. Au fond, ce qu'il s'agit d'expliquer, c'est comment et pourquoi l'homme de la rue a raison de continuer à nommer «ville» un objet dont la forme, le contenu et la signification n'ont cessé de changer au cours des siècles...

Le but de cet article est de proposer plusieurs pistes de réflexion tendant à formaliser une théorie évolutive des villes. L'objectif est de dépasser les difficultés des positions essentialistes ou structuralistes qui dominent la théorie urbaine. Faute de place, on se propose ici d'évoquer surtout la partie de la théorie qui concerne la notion de réseau urbain, ou de système de villes. On a observé depuis très longtemps l'universalité de la forme d'organisation, hiérarchique et spatiale, de ces systèmes, quelle que soit la région du monde, et sa pérennité dans la durée, quel que soit le moment de l'histoire. Parmi les pionniers 
mentionnons Reynaud (1841) cité par Robic (1982) et (1984), Auerbach (1913), Zipf (1949) et parmi les vérifications récentes Fletscher (1986) pour les traces archéologiques, de Vries (1984) pour des temps historiques à l'échelle de l'Europe et Moriconi-Ebrard (1993) pour la période contemporaine à l'échelle du monde. L'ambition de la théorie évolutive est d'expliquer la forte différenciation des villes par leur taille et leurs fonctions, c'est-à-dire la structure des réseaux urbains, sa persistance et son évolution dans le temps, à partir des relations entre les villes et entre les villes et leur environnement.

Une théorie évolutive n'est pas une théorie historique: il ne s'agit pas de prendre en compte toute l'histoire des villes, ni de reconstituer une genèse dans ce qu'elle a d'unique. Il ne s'agit pas de faire l'histoire entendue comme le chemin qu'on parcourt à reculons pour trouver une «explication» dans le récit de la biographie d'un lieu. Le projet n'est pas ici celui, descriptif, de la géographie historique. Il s'agit de formaliser l'évolution, le processus de la transformation historique d'entités spécifiques. L'enjeu est de montrer qu'un objet géographique peut s'interpréter comme la réalisation particulière, parmi un univers de réalisations possibles, d'un processus dynamique général. En simplifiant, en modélisant, on perd les notions d'unicité et de non-reproductibilité attachées aux objets historiques, mais on prend en compte l'irréversibilité de leur trajectoire particulière.

L'approche évolutive n'est encore qu'esquissée sur le plan épistémologique en géographie. Elle suppose d'analyser les processus qui intègrent la durée dans la définition même des objets géographiques. Plutôt que d'en donner une présentation directe, dont l'axiomatique reste encore à préciser, nous retraçons ici le cheminement qui a conduit à sa formulation dans le cas des systèmes de villes.

\section{Critique des explications statiques}

Dans la mesure où leur structure est facilement repérable et n'évolue que lentement, il n'est pas étonnant que les réseaux urbains, ou plus généralement les systèmes de peuplement, aient été formalisés d'abord par des théories statiques. Nous ne rappellerons pas ici toutes ces tentatives, dont les plus importantes sont la théorie des lieux centraux et la bien mal nommée «loi rangtaille» (pour des mises au point récentes, voir Beguin 1988, 1992a et b, Carrol 1982 et Guérin-Pace 1993). Des essais théoriques plus nouveaux concernant la ville et les modèles qui en dérivent sont construits, à partir d'une approche micro-économique, dans le cadre de la théorie des marchés et de l'équilibre (voir un état de ces questions dans Baumont et Huriot 1996 et
Derycke 1996). Ces essais sont le plus souvent menés par des économistes (par ex. Fujita 1989 et Fujita, Krugman, Mori 1994), mais parfois aussi par des géographes (Béguin 1996).

Nous voulons d'abord discuter un a priori, implicite dans ces travaux, selon lequel on pourrait déduire une explication suffisante de la ville, à partir des principes de la théorie économique générale, bien que celle-ci ait d'abord été conçue en dehors de toute référence spatiale. En effet, la ville est un objet complexe, dont l'approche économique ne saurait épuiser la définition (Camagni 1993, Pumain 1994). De plus, bien souvent, la confrontation avec les faits demeure une sérieuse pierre d'achoppement pour ces théories, dans la mesure où de nombreux concepts qu'elles utilisent sont inaccessibles à l'observation.

La tentation de la théorie «pure» (si tant est que l'on sache la définir) peut être forte, l'aspiration à une formalisation axiomatique et mathématique rigoureuse est sans doute légitime. Elle est toutefois insuffisante dans la mesure où, en sciences humaines, l'apport d'intelligibilité se heurte tôt ou tard à l'observation du monde. D'où mon plaidoyer pour une théorie peut-être moins ambitieuse mais qui ne néglige pas les allersretours avec l'observation. Le processus de construction théorique par cette méthode dite «rétroductive» est modeste. Il impose que la théorie permette de déduire des modèles qui soient compatibles avec des régularités statistiques observées qu'ils formalisent, il implique aussi dans la pratique de limiter les propositions théoriques efficaces aux domaines où un test, une mise à l'épreuve sont possibles.

Par ailleurs, l'économie spatiale est très largement statique: il est à cet égard symptomatique que l'Encyclopédie d'économie spatiale (Auray et al. 1994) ne comporte pas un seul chapitre sur la croissance, ou la dynamique des espaces... Les théories et les modèles statiques ont certes leur utilité. Certains permettent de faire des calculs et des projections à court terme, qui se révèlent souvent d'un grand intérêt. Le propos n'est donc pas d'éliminer ces outils irremplaçables. Il ne s'agit pas non plus d'incriminer leur incapacité à prendre en compte ou prévoir le changement, puisqu'ils ne sont pas faits pour cela. Il s'agit ici de remettre en cause leur statut explicatif.

\section{1. Économies d'agglomération ef lieux centraux}

La plupart des auteurs qui s'inspirent de la théorie microéconomique pour produire des théories urbaines justifient le regroupement spatial des agents économiques, qui sont censés maximiser leur utilité, par l'existence d' "économies d'agglomération», ou "économies d'échelle externes à la 
firme» (Catin 1994, p. 105). Une première difficulté provient de ce que ces fameuses "économies d'agglomération", souvent postulées, ne sont jamais mesurées. En outre, pour choisir leur localisation en fonction des économies d'agglomération, les agents ont besoin que la ville existe déjà, et la théorie ne dit pas comment elle a pu se développer. Une autre critique à adresser à ces théories est qu'elles postulent le plus souvent l'existence d'une ville isolée: les agents se regroupent sur la base de leurs intérêts, sans qu'il y ait un rapport nécessaire avec d'autres villes. Lorsqu'il s'agit d'expliquer les systèmes de villes, et de rendre compte de leur structure hiérarchique, les théories économiques supposent l'existence de rendements croissants avec la taille des villes. Par exemple, pour Fujita $e t$ al. (1994), l'hypothèse est que le système optimise à tout moment son fonctionnement en réalisant un équilibre entre demande de services et offre à l'échelon mésoscopique de la ville, tandis que les individus optimisent leur localisation en maximisant leur utilité. Les villes qui offrent des services diversifiés vont être plus attractives, et l'afflux de migrants va susciter à son tour l'accroissement de la variété de l'offre de services. Les grandes villes sont donc plus attractives et croissent plus vite que les petites! La théorie n'explique pas pourquoi il y a des grandes et des petites villes, pourquoi la hiérarchie des villes prend une forme particulière et la maintient au cours du temps.

La théorie des lieux centraux, dans sa formulation initiale, est une théorie statique, qui présuppose aussi l'ordre qu'elle prétend expliquer. Ainsi, il suffit de postuler l'existence de niveaux de biens et de services différents (ordonnés par leur plus ou moins grande rareté d'utilisation, d'où découlent des portées et des seuils d'apparition variables) pour déduire l'existence de niveaux hiérarchisés de centres urbains et leur espacement régulier (Beckman et MacPherson 1970). Plusieurs auteurs, dont H. Beguin (1979 et 1988) ont introduit des éléments stochastiques afin d'adapter la hiérarchie trop régulière des modèles à la continuité des dimensions urbaines observées. Ce faisant, on n'a pas expliqué comment s'effectue le lien entre le classement des différents biens et le classement des villes. Le regroupement des biens de même niveau dans chaque ville est postulé et n'est pas démontré par la théorie. La théorie n'indique pas non plus comment la hiérarchie urbaine ainsi constituée est susceptible d'évoluer. L'ouvrage de Christaller contient un chapitre de propositions à ce sujet, mais qui ne sont pas intégrées dans ses modèles, qui demeurent structuraux et statiques.

\subsection{Théories statiques ef équilibre}

Les théories statiques de la ville suscitent le scepticisme car elles utilisent, à l'échelon des individus, c'est-à-dire des acteurs dont le comportement est formalisé par les modèles, des processus qui seraient valables en tout lieu et surtout en tout temps. Elles négligent le caractère historique de l'objet ville et donc se contredisent dans la mesure où elles tentent d'expliquer avec des mécanismes d'aujourd'hui une entité réalisée sur la durée. Il est probable que si l'on appliquait les principes de ces théories en construisant aujourd'hui de toutes pièces une ville, celle-ci ne ressemblerait, non pas seulement dans son caractère unique, mais dans son principe, à aucune des villes connues. La forme actuelle est le produit d'une évolution longue, elle ne peut correspondre à l'optimisation instantanée de quelques facteurs. Les mécanismes imaginés en micro-économie pour expliquer l'agrégation des agents, les économies d'agglomération, ne tiennent pas compte de la durée nécessaire à leur action.

Le problème est que, derrière toutes les explications statiques de la taille et de l'espacement des villes, se profile l'idée d'un équilibre, qui serait commandé par un facteur déterminant: c'est une force, une «main invisible», une contrainte statistique. Que l'on «explique» la forme des réseaux urbains par l'action de tendances contradictoires à la concentration et à la dispersion (Zipf 1949), par la théorie micro-économique des marchés, (dont la centralité serait le principe spatialisé selon Berry 1967), par un principe de maximisation des interactions sociales (Claval 1982) ou par un processus statistique qui déduit une structure à partir d'une combinatoire dominée par un principe limitant ou optimisateur (maximisation de l'entropie, Curry 1964), ces trois formes d'explication dérivent en fait de la même hypothèse. Selon cette hypothèse, les configurations que nous observons à un moment donné résulteraient de l'optimisation d'un factcur contraignant, ou manifesteraient un équilibre entre des actions contraires. Fujita et al. (1994) considèrent comme une «règle naturelle» (sic) que «à chaque moment du temps, le système urbain doit être en équilibre spatial» (p. 21). Dans cette version récente, ils tentent de dynamiser leur modèle en partant d'un modèle statique d'équilibre et en imaginant le déplacement de ce point d'équilibre au cours du temps. Un tel modèle ne permet certainement pas, contrairement à ce qu'indiquent ses auteurs, de comprendre «l'émergence d'un système de villes».

L'observation de la dynamique des réseaux urbains sur le long terme n'apporte pas de preuves que celle-ci serait gouvernée par un facteur macroscopique contraignant, ni que ce que nous observons à un moment donné soit en équilibre. Au contraire, nous observons des modifications, importantes ou graduelles, dans les distributions, sans pouvoir préciser si les forces qui sont censées les produire ont changé. Dans une distribution géographique, on peut ainsi repérer une certaine dynamique en 
cours. Cette dynamique est produite par des interactions qui, si on les laissait s'exercer en l'état pendant un temps assez long, pourraient conduire à un équilibre modélisable. Mais toujours, dans la réalité, la forme et l'intensité des interactions se modifient avant que cet équilibre puisse être atteint. Imaginer que l'état actuel d'un système correspond à un équilibre conduit donc à supposer, pour en rendre compte en ces termes, un ensemble d'interactions qui en fait ne peuvent pas à elles seules expliquer la forme actuelle (Allen 1991). Il nous faut donc penser que c'est à la fois la configuration observée, et ses transformations, qui doivent être explicables par une même théorie. La théorie doit expliquer ensemble la structure du système $e t$ ses changements.

L'hypothèse, un peu tautologique, sur laquelle s'appuient les économistes pour justifier le regroupement des activités dans les villes, et qui suppose a priori l'existence d'économies d'agglomération, ne me paraît pas nécessaire pour expliquer l'inégalité des tailles des villes et leur évolution. On peut en rendre compte avec une théorie évolutive, en se passant de l'idée selon laquelle les grandes villes seraient plus productives, plus performantes sur le plan économique, que les petites, pour expliquer l'existence des réseaux urbains dans leur forme actuelle. En fait, la plus grande productivité des grandes villes, si tant est qu'elle soit vérifiée (Rousseau, Prudhomme 1993), les économies d'agglomération donc, pourraient être autant la conséquence que la cause du succès des grandes villes.

\section{Des modèles dynamiques pour la croissance des villes}

On dispose désormais d'un grand nombre d'observations relatives au changement dans les systèmes de villes, grâce aux bases de données historiques et comparatives (Bairoch 1996). Pour intégrer ces observations dans une théorie, on a commencé par effectuer des transferts de modèles depuis les sciences «dures», mathématiques ou statistiques, physique-chimie, qui proposent des modèles dynamiques. L'intérêt de ces modèles, si réducteurs soient-ils, est leur fonction de filtre: en autorisant la comparaison entre la dynamique des villes et celle d'autres systèmes, ils ont permis de souligner certaines spécificités du changement urbain et d'aller ensuite au-delà dans la modélisation.

Il est d'usage, dans de nombreux modèles, de se contenter d'une description simplifiée des systèmes de villes, qu'on ramène à des ensembles d'agrégats de population caractérisés par leur nombre d'habitants. La simplification, certes très réductrice, n'est pas sans signification, dans la mesure où la variable «taille démographique » est un excellent (et le meilleur) résumé de très nombreuses propriétés fonctionnelles des villes. Beaucoup de descripteurs quantitatifs, comme le nombre d'emplois, d'établissements ou de logements... mais aussi qualitatifs, comme la diversité ou la rareté des activités et la variété des populations présentes, sont corrélés à la taille des villes. C'est la principale «dimension», c'est-à-dire le facteur de différenciation essentiel, dans un système de villes (Reiner, Parr 1980). Connaitre la taille d'une ville apporte en général beaucoup d'informations sur elle, c'est-à-dire permet de faire un grand nombre de prédictions qui sont le plus souvent vérifiées. En outre, sur de très longues durées, la taille a le mérite de pouvoir être évaluée assez simplement, de façon comparable, et de garder une signification relativement homogène en termes de «succès» du sous-système qu'elle représente. En effet, le fait d'attirer et de maintenir une certaine quantité de résidents a été pendant longtemps le critère de la capacité d'une ville à assurer les moyens de subsistance de cette population (par la production locale et l'innovation mais aussi par un prélèvement forcé sur des campagnes ou d'autres villes, ou encore par l'imposition de termes plus ou moins inégaux dans un système d'échanges). Toutefois, cette mesure de la réussite urbaine gagnerait à être complétée, en particulier pour des comparaisons internationales contemporaines, par des évaluations de niveau de vie et de quantité de production, ou par des indicateurs de qualité de vie et d'environnement.

On s'en tiendra provisoirement, dans le cadre de cet article, à cette description du système par la taille des villes pour la partie formalisée de la théorie. Cela ne signifie pas qu'une théorie évolutive doive s'arrêter là. Il s'agit d'une première étape, et nous verrons d'ailleurs qu'une conceptualisation cohérente et formalisćc conduit à intégrer, même dans cette version simplifiée de la théorie, des aspects beaucoup plus complexes de la définition d'un système de villes.

\subsection{Le modèle de Gibrat}

L'approche la plus simple qui permette de dépasser le point de vue statique est celle qui fait des systèmes de villes le produit d'un processus stochastique de répartition spatiale de la croissance de la population. Il s'agit de transférer un modèle statistique pour la description dynamique d'un système de villes. Dans son ouvrage sur Les Inégalités économiques, Gibrat nous apprend que, dans un processus où les villes se développeraient en moyenne à la même vitesse, mais avec des fluctuations, des inégalités de croissance, il est banal, «normal», d'observer une forme particulière de distribution des tailles de villes.

Soit un ensemble de lieux habités donné dont la distribution des tailles est quelconque (ils peuvent même être tous égaux 
au début du processus) et dont on modélise l'évolution sur une durée longue (par exemple quelques siècles), faite de courts intervalles de temps assez nombreux. On suppose que, lors de chaque intervalle de temps (par exemple une année, ou dix ans), la population $P_{\mathrm{i}}$ de chacun s'accroit, en moyenne (mais avec des fluctuations $e_{i}$ ) d'une quantité $d P_{i}$ qui est proportionnelle à la population $P_{i}$ (et qui est faible devant $P_{i}$ ).

$$
\mathrm{dP}_{\mathrm{i}}=r \mathrm{P}_{\mathrm{i}}+\mathrm{e}_{\mathrm{i}}
$$

Cela revient au même de dire que le taux de variation relative de la population $d \mathrm{P}_{\mathrm{i}} / \mathrm{P}_{\mathrm{i}}$ (c'est la mesure qui est en général exprimée en \% et utilisée pour caractériser et comparer la croissance des villes ou des régions) est le même en moyenne pour tous les lieux habités, et donc indépendant de leur taille au début de chaque intervalle de temps. Si, de plus, ces taux se redistribuent à chaque intervalle de temps entre les lieux habités d'une manière indépendante de celle de la période précédente, le processus de croissance ainsi défini aboutit toujours à une forme de distribution de taille des lieux habités qui est une distribution lognormale, très dissymétrique, avec un grand nombre de villages et de petites villes et une diminution géométrique du nombre des villes quand on considère des tailles de plus en plus grandes. On peut même prévoir (en probabilité) de combien l'inégalité des tailles de villes peut augmenter, si l'on connaît le taux de croissance et sa variance.

Avec ce processus, il n'est pas besoin de supposer que les villes sont d'autant plus attractives que leur taille est grande pour obtenir une distribution de tailles très inégale, comportant un petit nombre de très grandes villes (on peut donc se passer d'une théorie comme celle des économies d'agglomération). En outre, ce modèle est «testable», puisque l'on peut étudier de façon empirique les distributions statistiques des taux de croissance des villes par intervalles de temps sur des périodes longues, et il a été abondamment vérifié dans des pays et à des époques différents (par ex. Robson 1973, Pumain 1982, de Vries 1984, Guérin-Pace 1993, Moriconi-Ebrard 1993). On tient donc une «explication» plus satisfaisante de la forme des distributions de tailles de villes quand on en fait le produit, la conséquence d'un modèle dynamique de répartition de la croissance de la population des villes que quand on se contentait de la voir exprimer un équilibre statique. Par rapport à ces interprétations, le modèle de Gibrat est en effet le seul qui permette de comprendre pourquoi cette forme d'organisation se maintient au cours du temps, et de déduire une concentration progressive de la population dans des entités de plus en plus grandes et aux dimensions de plus en plus contrastées, ce qui est conforme aux observations. Le modèle donne ainsi une «explication» de la différenciation progressive de taille entre des villes qui participent de la même évolution, mais avec un inégal succès produit par la répétition «accidentelle» (ou aléatoire, au sens où elles ne sont pas déterminées a priori par le modèle) en certains lieux, d'accroissements infinitésimaux plutôt supérieurs ou plutôt inférieurs à ceux que réalisent l'ensemble des autres villes. En première approximation, le modèle de Gibrat, dans sa simplicité, constitue donc à la fois un bon résumé de la croissance d'un système de villes et une explication de sa structure hiérarchique. Il est utilisable pour faire des prévisions de l'évolution de la taille des villes sur des durées de l'ordre de quelques décennies.

Toutefois, une théorie des systèmes de villes ne peut se réduire à la formulation de ce modèle purement stochastique, en particulier si l'on souhaite intégrer dans la théorie l'observation récurrente de certains écarts au modèle de Gibrat: d'une part, les taux de croissance des villes sont presque toujours, sur le long terme, très légèrement corrélés positivement à la taille des villes, et d'autre part il arrive qu'ils soient autocorrélés dans le temps, sur des durées de quelques décennies (Robson, 1973, Pumain, 1982, Guérin-Pace, 1993). Par ailleurs, les villes «primatiales», ou les deux ou trois «métropoles» des hiérarchies urbaines nationales, dont la taille apparaît «disproportionnée» par rapport au reste du système de villes, sont en fait des agglomérations qui se sont développées pendant plusieurs décennies systématiquement plus vite que le reste du réseau urbain (Moriconi-Ebrard 1993, Pumain, Moriconi-Ebrard 1997). Le modèle doit donc être corrigé et complété pour tenir compte de ces «anomalies», qui ne sont définies comme telles que par rapport au processus stochastique simple, mais qui sont en fait révélatrices de processus spécifiques à l'évolution du système des villes. On est alors conduit à formaliser explicitement des relations d'interdépendance entre les villes, et donc à passer de la formalisation d'un ensemble de villes à celle d'un système de villes. L'idée d'une interdépendance entre les villes demeure en effet implicite dans le modèle de Gibrat, elle ne se réalise que par l'hypothèse que les taux de croissance des villes obéissent à une distribution de même moyenne et d'une variance donnée: il faut donc supposer qu'elles appartiennent au même «système». Cette hypothèse ne figure pas en tant que telle dans le modèle statistique, c'est lors du transfert aux villes de ce modèle qu'on la fait logiquement intervenir comme l'expression des échanges d'informations et de phénomènes de concurrence entre les villes.

Une autre observation va d'ailleurs obliger à préciser un autre processus, qui est celui de la sélection des entités qui vont devenir des villes parmi l'ensemble de tous les lieux habités. La dynamique que décrit Gibrat s'applique en effet non seulement aux villes, mais plus généralement à l'ensemble d'un système de peuplement. Or, il est patent que cette dynamique tend à produire non pas une, mais deux distributions lognormales, caractérisées par des degrés inégaux de contraste entre les dimensions de leurs éléments (d'autres auteurs interprètent la 
même observation par un modèle de type «fractal parabolique», qui ne constitue cependant qu'une description statique, cf. Laherrère 1995). De notre point de vue, cela implique que deux sous-ensembles de lieux habités aient été durablement "sélectionnés" pour une dynamique de croissance et une de déclin, comme cela a été le cas pour les villes et les communes rurales en France depuis près de deux siècles (Pumain 1995, p437). La distinction entre villes et villages va ainsi conduire à intégrer dans la théorie évolutive des considérations qualitatives relatives aux fonctions des agrégats de peuplement, et aussi la tendance historique de la «transition urbaine».

\subsection{Une dynamique de l'inferdépendance}

La communication, l'échange, l'interdépendance avec des entités de même nature sont des propriétés qui paraissent liées à une définition géographique du concept de ville. En ce sens, un géographe peut difficilement se satisfaire de la définition (présentée comme une «non-définition»!) proposée par H. Beguin (1996), qui insiste sur le concept de densité, mais n'articule pas bien les deux niveaux repérables de l'organisation urbaine, la ville et le système des villes, et surtout n'intègre pas la dimension «écologique», l'explication «verticale» et non plus seulement «spatiale» ou «horizontale» de l'objet ville. La ville ne peut pas être dissociée du système des villes, et l'échange, quelles qu'en soient la nature et la forme, donc les connexions à longue distance, font partie intégrante de la fonction urbaine, de sa spécificité. Selon Henri Reymond (1981), la possibilité d'exploiter une situation, les ressources de plusieurs sites, est ce qui fait l'originalité de la ville par rapport aux autres formes de peuplement (village, exploitation minière) qui exploitent des ressources locales, tirées d'un site unique. Cette conception est compatible avec les enseignements de l'histoire et de l'archéologie quant à la naissance des villes. Selon P. Bairoch, en effet, «l'existence d'un centre urbain présuppose non seulement un surplus agricole, mais aussi la possibilité d'échanger ce surplus» (1996, p.19).

H. Reymond justifie sa définition en empruntant à l'écologie le concept de facteur limitant, selon lequel à un moment donné les ressources accessibles à une population localisée à un endroit donné deviennent une limite à son développement: «Le système d'habitat urbain est l'organisme écologique de base qui, parmi tous ceux que l'espèce humaine a inventés, peut annihiler de manière ubiquiste l'ensemble des facteurs limitants d'un site, quelles que soient ses ressources propres. Les villes ont réussi à faire face aux incertitudes nées des facteurs limitants locaux, à réguler ces difficultés locales, en développant des liaisons multiples, des réseaux sur lesquels les vitesses sont de plus en plus grandes. Les sites ainsi mis en correspondance peuvent recevoir des populations qui dépassent de beaucoup ce que permettrait le site initial. Cette évolution n'est pas terminée, et l'on continue d'inventer l'outil que deviennent ces villes». (communication orale, 1995).

La cohérence de l'évolution de villes interdépendantes a été pressentie depuis longtemps, par exemple par J. Reynaud lorsqu'il lance le concept de "système général des villes» (1841). En 1964, B. Berry, avec son expression fameuse «cities as systems within systems of cities», a formalisé dans le cadre de la théorie des systèmes la relation entre le niveau d'organisation et d'observation constitué par la ville, considérée comme ensemble de quartiers, d'institutions ou d'acteurs en relations systémiques, et le système des villes, organisé par les principes de la théorie des lieux centraux. Dans son ouvrage de 1977 , A. Pred insiste sur l'aspect dynamique de la notion de système de villes en lui appliquant une définition très générale: «un ensemble, national ou régional, de villes interdépendantes, de telle façon que tout changement significatif dans les activités économiques, la structure professionnelle, le revenu ou la population d'une des villes entraine directement ou indirectement quelque modification dans les activités économiques, la structure professionnelle, le revenu ou la population d'un ou de plusieurs autres éléments de l'ensemble».

Des recherches empiriques ultérieures portant sur les modalités de l'évolution des systèmes de villes ont montré l'intérêt d'une interprétation de la dynamique de ces systèmes dans le cadre de la théorie de l'auto-organisation. Cette formalisation permet de préciser la définition d'A. Pred et en particulier la notion de «changement significatif». En effet, ce n'est pas n'importe quelle transformation locale qui est susceptible d'affecter les autres villes du système: un très grand nombre des changements, même «significatifs", qui interviennent dans les villes au cours d'une durée de quelques années ou de quelques décennies (dans la morphologie urbaine, les structures démographiques, sociales ou économiques...) sont sans effet sur la structure du système, sur l'état de la différenciation interurbaine, car ils sont communs à toutes les villes (Pumain, SaintJulien 1978, Sanders 1992). D'autres changements, propres à une ville, ne remettent pas non plus en cause la structure du système, car ils ne sont pas suffisamment persistants d'une période à une autre, ce sont de simples fluctuations qui n'altèrent pas la place relative des villes dans le système. Le changement de structure intervient lorsque l'une de ces fluctuations persiste et s'amplifie au point de modifier durablement cette position, le plus souvent pour plusieurs villes à la fois, associées par exemple au développement d'une spécialisation nouvelle. On constate alors une bifurcation dans l'histoire du système. La plupart des fluctuations restent sans effet si elles se produisent 
lorsque le système est sur une trajectoire stable (Pumain 1987, Pumain et al. 1989). Une bifurcation peut aussi résulter d'une perturbation d'origine extérieure au système considéré.

\subsection{Les modèles issus des théories de l'auto-organisation}

C'est donc l'observation des modalités de l'évolution de villes interdépendantes qui a suggéré l'emprunt et le transfert de modèles issus de la chimie ou de la physique à la géographie. L'analogie avec des systèmes physiques, par la théorie des structures dissipatives (Allen 1978) ou par celle de la synergétique (Haken, 1977, Sanders 1992), invite à formaliser la dynamique des systèmes comme produite par les interactions entre des éléments microscopiques qui forment des structures repérables au niveau macroscopique, en sachant par ailleurs que le système est "ouvert»: s'il s'agit d'un système physique, cela signifie qu'il reçoit de «l'extérieur» un apport d' «énergie» qui facilite son «auto-organisation». L'équivalent de l'apport énergétique, lorsque le modèle est transposé aux systèmes humains, est assimilé à l'innovation, ou à une tendance à la croissance du système, qui constitue le «moteur» dc sa dynamiquc. La notion d'auto-organisation, transposée à ces systèmes, suggère que, pour expliquer leur structuration selon une architecture identifiable parce que persistante, il n'y a pas un opérateur de réseau, ni une finalité politique ou économique explicite, qu'il serait possible de décrire en termes d'une contrainte à respecter ou d'une fonction à optimiser. La structure est le produit involontaire d'interactions multiples entre des acteurs très nombreux. Chacun agit selon ses finalités et ses stratégies propres, mais il n'est pas nécessaire de connaître chacune en détail pour prédire (ou simuler) l'architecture générale du système. La multiplicité des desseins individuels peut ainsi se réduire à quelques paramètres et quelques équations décrivant l'évolution du système à un niveau macroscopique : c'est ce que permettent en principe les modèles probabilistes issus de la synergétique ou des théories de l'auto-organisation, qui résument dans une "équation mâ̂tresse» l'effet global des interactions entre des particules dont il est matériellement impossible de connaître (ou de prévoir) l'état et la position de chacune dans le système. La notion d'événement aléatoire est ainsi liée à l'existence de plusieurs niveaux d'observation dans le système: «les phénomènes microscopiques influencent le déroulement du phénomène macroscopique, tandis que c'est la loi de celui-ci qui agrège les phénomènes microscopiques, ou, en d'autres termes, leur «impose» son "point de vue». Les lois propres des deux types de phénomènes ne sont pas en général des «lois du hasard», celles-ci sont seulement les lois de leur interaction» (Bonitzer 1984).
Les systèmes auto-organisés ne sont pas en équilibre à un moment donné, ils se transforment sous l'action de processus dynamiques internes et externes. Leur structure est le produit de cette évolution. Les modalités du changement dans les villes ou dans les systèmes de villes ressemblent à celles que décrivent les théories physiques de l'auto-organisation. On observe à la fois une persistance ou une transformation lente de la structure du système (état macrogéographique du système des villes décrit par exemple par la forme de la distribution des tailles des villes, les principaux facteurs de différenciation des activités et des compositions sociales des villes...) et de très grandes fluctuations dans la situation relative de ses éléments: au niveau méso-géographique, c'est le passage rapide que décrit chaque ville, entre des «états» de croissance, stagnation, décroissance, ou des changements de rang dans la hiérarchie des tailles sur la moyenne durée, ou bien encore les modifications décennales de leurs profils socio-économiques, qui sont tantôt en avance, tantôt en phase ou en retard sur une transformation commune (Pumain, Saint-Julien 1978). Les fluctuations (ou passage des éléments d'un état à un autre) sont encore plus évidentes si l'on considère le niveau d'observation le plus "microscopique» pour l'investigation géographique, c'est-à-dire celui des individus, ménages ou entreprises, qui résident dans les villes, à l'échelle de temps d'une année (mobilités résidentielles et professionnelles, créations et disparitions d'entreprises) comme à celle du remplacement des générations.

Des modèles dynamiques des systèmes de villes ont été élaborés dans ce contexte, et explicitent dans leur formalisation certaines interdépendances entre les éléments du système, par exemple la concurrence pour l'acquisition d'activités ou l'attraction des populations. Des modèles dynamiques permettent de simuler l'évolution d'un ensemble de lieux centraux à partir des croissances et des échanges de population et d'emploi entre les villes d'une région (Allen, Sanglier 1979, White 1977). D'autres modèles simulent l'évolution des tailles des villes à partir des migrations des individus entre les villes (Weidlich, Haag 1988, Sanders 1992). On a pu relier ces modèles à la structure macroscopique du système des villes, par exemple en démontrant que, sous certaines hypothèses, la distribution de la taille des villes constituait un attracteur pour un modèle dynamique de type synergétique des migrations interurbaines (Pumain, Haag 1994).

Plusieurs difficultés sont apparues lors du transfert des ces modèles et de leur application. D'une manière générale, la description de la dynamique des systèmes géographiques à l'aide de modèles élaborés pour des systèmes physiques a l'intérêt de permettre une formalisation satisfaisante des liens entre deux niveaux d'observation de ces systèmes. Toutefois, 
les mécanismes du changement paraissent parfois de nature sensiblement différente, les systèmes géographiques de moyenne échelle connaissant plutôt moins de bifurcations que ce que les modèles ont tendance à reproduire (Sanders 1993). Peut-être aussi la notion de dynamique employée dans ces modèles simplifie-t-elle outrancièrement les temporalités urbaines (Lepetit, Pumain 1993). L'explication de type dynamique atteint aussi rapidement ses limites. Dans les modèles dynamiques de lieux centraux, on est obligé d'introduire de façon exogène des niveaux de fonctions supérieurs pour simuler la construction progressive d'une hiérarchie urbaine, ce qui revient, comme pour les modèles statiques, à une explication tautologique. À propos du modèle synergétique des migrations interurbaines, il est aussi douteux que l'on puisse trouver dans la théorie individuelle de la décision de migrer, une explication de la genèse des systèmes de villes qui soit suffisante. Ces modèles résument en effet l'utilité individuelle de la migration par un paramètre qui est en fait très largement une attractivité globale attribuée à la ville de destination et fortement dépendante de la taille de celle-ci. Même si cette attractivité se calcule à partir du schéma des migrations observées à une époque donnée, on retrouve l'objection d'explication tautologique déjà signalée à propos des économies d'agglomération. En outre, l'observation empirique des migrations interurbaines a montré à maintes reprises la forte dépendance des flux migratoires à l'égard de l'état antérieur de la différenciation des villes (en taille et en fonctions), qui en ferait un instrument «passif» d'ajustement à une évolution en cours du système des villes, plutôt qu'un moteur de cette évolution (Greenwood 1981, Pumain 1980, Pumain, Saint-Julien 1989), cela bien évidemment en dehors des afflux exceptionnels (immigration massive, réfugiés...) qui eux seraient susceptibles de faire bouger le système.

Une objection plus générale, et peut-être plus décisive, est en effet que les modèles dynamiques décrivent comment s'opère une évolution plutôt que pourquoi elle se fait. Si le mécanisme essentiel qui régit la forme du système des villes est celui de la concurrence entre les unités géographiques qui le composent, pour capter et accumuler le produit de ressources diverses, et si le jeu de cette concurrence est permis par la circulation de l'information entre des unités qui se mettent ainsi en réseau, il reste que le moteur de l'évolution est la création continue de nouveauté et de richesses dans le système. Or l'émergence de l'innovation demeure une pierre d'achoppement de la modélisation dynamique, que résolvent mal même les tentatives de simulation les plus sophistiquées (Allen 1991).

Cette difficulté à prendre en compte dans des modèles mathématiques la création, le dépassement récurrent des limites d'une dynamique, qui est la caractéristique des processus évolutifs sociaux, explique que les modèles fondés sur une analogie biologique n'aient guère plus de pertinence que les transferts de modèles physiques. La transposition des modèles proie-prédateur de Volterra-Lotka à l'étude de la concurrence entre des régions ou des villes (Dendrinos et Mullaly 1985) n'est possible que si l'on se limite à l'observation de variations relatives, autrement dit si on réduit la dynamique des villes à une lutte pour accaparer des parts de marché (modèle de «substitution technologique» de Marchetti 1986). Or c'est ignorer d'une part la remise en cause permanente, par les sociétés humaines, du facteur limitant des modèles de concurrence écologique du type Volterra-Lotka et d'autre part la réutilisation toujours possible des territoires provisoirement abandonnés pour d'autres usages lors d'un nouveau cycle d'innovations, qui rend inopérante l'idée d'une analogie avec la substitution technologique entre des produits.

On touche ici aux insuffisances des analogies physique ou biologique lors de leur transfert à une théorie des systèmes de villes. Bien qu'ils prévoient deux processus possibles d'intervention du changement dans la structure des systèmes, par bifurcation du fait de l'amplification d'une fluctuation interne ou de l'irruption d'une perturbation externe, il est très difficile de prendre en compte dans les modèles inspirés des systèmes physiques le phénomène essentiel de l'innovation. Non seulement celle-ci dans les systèmes de villes est très largement endogène, mais, par le renouvellement permanent qu'elle induit, elle prend une importance primordiale dans la genèse de la structure du système, par diversification et complexification de ses éléments. Il paraît peu pertinent de ne la considérer que comme une fluctuation ou une perturbation externe. Les économistes soucieux de remplacer la théorie de l'équilibre général par des considérations évolutionnistes s'attachent d'ailleurs à préciser ce processus.

Certains auteurs ont aussi proposé d'incorporer à la théorie, non seulement des aspects de dynamique physique, mais encore des processus évolutifs inspirés des théories du vivant. Pour P. Allen (1991), les villes appartiennent à des types de systèmes dans lesquels du nouveau se crée. Cette nouveauté apparaît dans les systèmes, non pas en vertu de l'optimisation de leur fonctionnement d'un moment, mais à la faveur des errements et des «découvertes» ou des «inventions» de déviants. C'est en fait la notion de variété de la théorie de l'évolution biologique qui reçoit ici une interprétation en termes sociaux. Les modèles issus de cette théorie requièrent de très grands ordinateurs pour simuler l'émergence endogène de la nouveauté. Il n'est pas sûr cependant que l'innovation sociale, avec les processus d'apprentissage et de concertation qu'elle implique, puisse se modéliser tout à fait comme un phénomène d'ordre biologique. 
Il faudra sans doute faire intervenir, dans les modélisations futures, des processus cognitifs, qui pourraient d'une part intervenir dans l'apparition et dans la sélection de l'innovation, et d'autre part intervenir comme des régulateurs supplémentaires dans l'évolution. On expliquerait ainsi que les systèmes urbains soient moins susceptibles de bifurcations ou de comportements «chaotiques» que d'autres types de systèmes évolutifs.

\section{De la dynamique à la théorie évolutive}

Toutes ces tentatives contribuent cependant à enrichir la conception de ce que devrait être une véritable théorie évolutive des systèmes de villes. Une théorie évolutive doit admettre que l'objet dont elle s'occupe, qui se transforme au cours du temps, procède de ses formes antérieures, tout en acquérant des propriétés nouvelles. La théorie indique quelles sont les tendances générales suivies par ces changements, quelle est la direction de l'évolution, ce qui permet éventuellement d'étayer des prévisions. Il serait prématuré de prétendre qu'une formalisation achevée est possible, mais nous suggérons quelques réflexions qui pourraient être utiles à sa construction.

\subsection{La notion de système de villes}

Un réseau urbain ou système de villes peut être défini simplement comme un sous-ensemble d'un système de peuplement. Celui-ci comprend l'ensemble des lieux habités d'un même territoire, hameaux, villages ou villes, ainsi que des relations (à préciser par la suite) entre ces lieux habités d'une part et avec leur environnement d'autre part. L'appartenance à un même territoire garantit une certaine cohérence de fonctionnement du système, en assurant l'homogénéité relative des conditions de communication entre ses éléments. Le territoire est ici précisément défini selon une acception politique ou culturelle, c'est une portion contiguë de la surface de la terre appropriée ou contrôlée par un groupe. La notion de territoire suppose aussi un certain degré de fermeture (les "frontières" réduisant l'intensité des interactions entre les lieux appartenant à des territoires différents) qui justifie l'identification d'un «système». Toutefois, il faut noter que les systèmes de villes continuent à se développer, même lorsque les territoires politiques se modifient et se recomposent. Par ailleurs, les organisations politico-administratives de territoires particuliers sont susceptibles de produire des variantes spécifiques du modèle général de système de villes, lorsqu'ils sont demeurés stables sur de très longues périodes. Enfin, à des degrés divers, il est certain que les villes, surtout dans les niveaux supérieurs de la hiérarchie, se jouent des frontières territoriales. La portée des relations urbaines dépendant forte- ment des tailles des villes, le territoire de référence devrait être à géométrie variable selon le niveau hiérarchique considéré. En fait, la notion de système appliquée aux villes trouve là ses limites, une autre métaphore plus adaptée devrait être recherchée. Elle garde cependant dans les modélisations une certaine opérationalité, nous l'emploierons donc avec réserve en attendant mieux.

Le temps de l'évolution est constructeur de complexité. La perspective évolutive amène à intégrer, dans la définition même des objets, la ville et le système des villes, leurs temporalités spécifiques. Cela ne peut se faire sans adopter une conception relative de l'espace géographique.

\subsection{Espace relatif et entités géographiques}

Dans la pratique de l'activité des géographes, deux conceptions de l'espace coexistent. L'une, la plus courante, l'assimile à un référentiel fixe, qui sert de support, de cadre pour l'analyse des activités humaines qui s'y répartissent (Beguin, Thisse 1979). Ce référentiel possède des propriétés géométriques simples, même s'il donne une représentation approximative et déformée de la surface de la terre, par exemple dans les constructions cartographiques. L'autre conception définit l'espace à partir des relations qui s'établissent entre les lieux. Bien souvent, dans la mesure où ces relations ne sont pas distribuées de façon homogène, ni isotrope, ni continue, l'espace «relatif» ainsi obtenu n'est pas susceptible de représentations simples. En revanche, la référence à cet espace relatif est essentielle pour comprendre l'émergence de ce que l'on dénomme, dans un espace absolu, des «structures spatiales», des objets géographiques repérables et identifiables.

Bien que la théorie n'en soit encore qu'esquissée, une perspective évolutive montrerait comment ces entités géographiques, qui peuvent prendre la forme de villages, finages, villes, territoires, réseaux, sont construites par intégration, au cours du temps, de relations entre les lieux. Les fréquentations, les échanges, développent la diffusion et l'imitation des pratiques, l'interconnaissance et les affinités. Ces relations progressivement induisent les ressemblances qui font les paysages et les régions homogènes, et les appartenances qui définissent les territoires ou les réseaux. Elles suscitent les accumulations qui renforcent le pouvoir d'attraction de certains lieux. Ces types de constructions s'imbriquent à différentes échelles pour former l'espace géographique. Philbrick (1957) en a donné une description statique. C. Grasland (1994), par exemple, tente d'en faire une analyse formelle. Il reste à en produire une théorie dynamique. 


\subsection{Les villes construisent deux espaces}

Les modèles dynamiques formalisant les villes comme des systèmes auto-organisés ont utilisé jusqu'ici la représentation d'un espace absolu, dans lequel les objets villes se localisent et sont reliés entre eux par des flux (matériels, de personnes et d'information) pour former un système. Pour passer d'une théorie dynamique à une théorie évolutive, il est nécessaire de concevoir un espace relatif, qui soit défini par ces relations et ces flux. C'est alors qu'on est amené à distinguer les deux niveaux d'observation du phénomène urbain que sont la ville et le système des villes. D'une part, les deux niveaux d'observation du fait urbain correspondent à deux types de territoires, dont les échelles relationnelles sont différentes, et d'autre part la formation et l'histoire des systèmes de villes s'éclairent par les transformations de cet espace relatif (Pumain 1995b).

À un moment donné, le système des villes, ou plus généralement le système de peuplement, permet l'articulation d'entités territoriales à des échelles géographiques différentes, en mettant en relation des niveaux d'organisation qui n'ont pas les mêmes ordres de grandeur spatio-temporels. Le système de peuplement permet ainsi de gérer la coexistence d'au moins deux niveaux principaux. Le premier est celui du village ou de la ville en tant que territoire de fréquentation quotidienne. Selon les époques, la composition des déplacements internes a pu varier sensiblement, en particulier en fonction du degré de spécialisation des lieux entre la résidence, le travail, la chalandise, la promenade... Dans l'ensemble, le temps moyen consenti à ces déplacements quotidiens, qu'il faut ajouter à celui des autres activités effectuées dans le cadre d'une journée, demeure relativement invariant, il se situe aux environs d'une heure, parfois entre une et deux heures. L'extension spatiale des territoires du quotidien reste donc très fortement contrainte par la durée des déplacements internes, dont la vitesse, en particulier dans les grandes villes, est elle-même ralentie par la forte densité (par exemple, la vitesse moyenne de circulation automobile à Paris serait aujourd'hui de $18 \mathrm{~km} / \mathrm{h}$ ).

Les fonctions principales de ce territoire du quotidien que représente chaque noyau du système de peuplement sont l'habitat et la production (de biens et de services). Ces deux fonctions majeures ne sont cependant plus, dans la plupart des cas, régulées localement. Pour l'essentiel, les déterminants de leur évolution se situent ailleurs, dans les nœuds des réseaux de pouvoir où s'effectuent les prises de décision. Dans ces réseaux, certains nœuds de pouvoir sont clairement identifiés, par une capitale politique ou administrative, par le siège d'une grande entreprise multinationale, mais le plus souvent c'est par des réseaux multiples de négociation, de concurrence ou de complémentarité, que se règlent les modalités des prélèvements ou des échanges, matériels, de personnes ou d'information, qui assurent le fonctionnement des territoires du niveau d'organisation supérieur, d'échelle régionale ou nationale, voire supranationale.

C'est le système des villes qui fait fonctionner ces réseaux. Les modalités de la circulation en vigueur dans cet autre niveau de l'organisation territoriale règlent la trame spatiale du système, définie par l'espacement et la taille des villes. À ce niveau, c'est à peu près la durée d'une journée de voyage qui détermine l'espacement entre les principaux nœuds. Cette durée est admise en moyenne pour effectuer les étapes d'un voyage (Reclus 1895) ou un aller-retour en vue de mener des négociations relativement fréquentes dans des lieux qui ne sont cependant pas visités chaque jour (Cauvin, Reymond, Schaub 1989), dans les réseaux potentiels d'échange entre sièges sociaux et filiales, entre la grande entreprise et ses clients, entre une capitale et ses principaux relais administratifs. Quoique peu précis, cet ordre de grandeur d'une journée assure une possibilité d'extension spatiale bien plus grande pour les territoires du pouvoir que pour ceux du quotidien. En effet, non seulement la durée qui règle l'espacement des næuds dans un même territoire, mais aussi la vitesse de circulation entre ces nœuds, pour un moment donné de l'histoire des techniques de transport, sont généralement bien plus grandes que celles qui sont praticables à l'intérieur de chaque nœud (Orfeuil 1993, Dupuy 1995).

La définition d'un espace relatif pour représenter les possibilités relationnelles, à l'intérieur des villes d'une part, entre les villes d'autre part, justifie l'intérêt porté à la géométrie fractale pour étudier ces ohjets (Arlinghaus 1985, Frankhauser 1993, Batty, Longley 1994). L'espace relationnel, fortement organisé par des gradients, possède une structure de type fractal. La notion de fractalité a le mérite de préciser, pour la description des structures urbaines, un nouveau modèle de référence, qui cesse d'être celui d'une occupation homogène de l'espace comme dans le calcul de la densité. Elle devient l'expression directe de la manière anthropologique d'occuper l'espace, en incorporant dans la description des objets urbains la forme engendrée par la pratique de la centralité. Celle-ci fait que les densités urbaines (du bâti, de la population, des activités, des déplacements...) ne sont pas distribuées dans l'espace de façon homogène mais varient systématiquement en fonction de leur position relative, par rapport à un ou plusieurs centres, selon une dilution ou une fragmentation du centre vers la périphérie. Un modèle fractal est donc mieux adapté qu'une référence euclidienne pour analyser les propriétés des systèmes urbains et faire surgir par différence les spécificités propres à chacun. Les 
premières expériences menées sur les villes et les réseaux urbains montrent la bonne adéquation des modèles fractals aux différents niveaux d'analyse (Le Bras 1996). Certains soulignent en outre une césure assez nette entre la fractalité des espaces internes aux villes et entre les villes (François et al. 1995), qui confirmerait l'identification de deux niveaux de structuration spatio-temporelle par les villes. La représentation par les fractales pallie d'ailleurs l'insuffisance des représentations spatiales de la géométrie des systèmes de villes par les modèles de Christaller, qui négligent les différentiels de densités créés par la présence des villes. Approximativement schématisée par W. Isard, cette "déformation » infligée aux modèles spatiaux des lieux centraux avait, selon M.-C. Robic, déjà été pressentie par J.Reynaud qui suggérait une «courbure de l'espace» à proximité des villes ou des voies de transport.

\subsection{Vers une théorie de l'évolution des systèmes de peuplement}

Concevoir le système des villes comme réalisant l'articulation entre deux ordres de grandeur des relations spatio-temporelles implique qu'on ne se contente pas d'une théorie formelle statique. La structure actuelle des systèmes urbains ne représente en effet ni un état d'équilibre, ni un optimum établi sous l'effet d'une contrainte quelconque à un moment donné. La forme de la trame des villes, leur dimension et leur espacement, résultent de processus plus ou moins continus d'adaptation des villes à une évolution du dimensionnement de l'espace par les vitesses de circulation, et d'ajustement de leur contenu à l'évolution sociale, fonctionnelle et technique.

La théorie évolutive replace la structure actuelle d'un système de villes dans l'histoire générale des systèmes de peuplement. Celle-ci est marquée profondément par le processus de la transition urbaine. On désigne ainsi la transformation universelle qui accompagne le passage d'une économie agraire, exploitant principalement des ressources locales, à une économie industrielle et tertiaire, fondée sur les échanges à longue portée. Dans les pays de peuplement ancien, comme l'Europe ou l'Asie, ce sont les mêmes sites, les mêmes noyaux de peuplement, qui ont été réutilisés par les systèmes économiques et politiques régnant successivement sur les territoires. La création ex nihilo de nouveaux sites de peuplement, de villes nouvelles, est un processus très marginal par rapport à l'écrasante majorité des processus d'adaptation de lieux déjà habités à de nouvelles fonctions. Dans les pays de peuplement plus récent, une première phase de l'évolution s'est aussi traduite par une diffusion spatiale suivant le front de colonisation, avec une création de nouveaux sites, mais les modalités de l'évolution sont devenues semblables à celles des pays anciennement peuplés dès lors que le territoire accessible a été entièrement occupé.

La principale caractéristique dynamique des systèmes de peuplement urbains semble être la concurrence entre leurs éléments, pour la captation et la création de richesses, par l'échange, l'innovation (anticipation) et l'adoption (imitation) des innovations. Cette forme d'évolution est permise par la très forte connexité du système, qui assure la circulation de l'information. Lorsqu'un changement apparaît dans une ville, il est très vite imité et adopté dans les autres villes. Le pouvoir d'adaptation au changement est lié à la position relative des villes dans le système en termes de ressources, liées à leur site ou accumulées, et de très nombreux auteurs ont illustré le lien entre vitesse d'adaptation au changement et accumulation antérieure (résumée par la taille des villes) en décrivant un processus de diffusion hiérarchique des innovations dans les systèmes urbains (Pred 1966 et 1973). Loin de restreindre le volume de la population et des activités urbaines comme dans un modèle biologique d'interaction proie-prédateur, la concurrence interurbaine n'est pas un jeu à somme nulle et, parce qu'elle suscite l'innovation, conduit à repousser continuellement les facteurs susceplibles de limiter l'expansion des villes. Elle est à l'origine du processus de division sociale du travail qui exprime cette quête et suscite de nouvelles spécialisations urbaines, et qui doit donc pouvoir être conçu de façon endogène dans un modèle. La concurrence entre les villes pour la captation des ressources éloignées et du produit des innovations conduit, en un temps relativement court (dépendant de la vitesse des communications du moment), à un processus de croissance et de transformation de l'ensemble des villes dans le territoire considćrć. Cette expansion quantitative et qualitative généralisée apparente l'urbanisation à un processus de diffusion-expansion, qui atteint toutes les parties du système des villes. Ce processus est stochastique, fait de petits ajustements qui adaptent (ou corrigent) en permanence la place des villes dans le système, chacune tendant à conserver sa position relative. Ce même processus rend compte de l'organisation hiérarchique du système, telle que la décrit le modèle de Gibrat.

Les anticipations ou les imitations auxquelles se livrent les acteurs urbains sont cependant des processus non linéaires qui peuvent entraîner des bifurcations, c'est-à-dire des modifications de la structure définie par les positions relatives des villes dans le système. C'est ainsi que, dans les périodes de mise en place des systèmes de peuplement, de bouleversement de l'organisation politique des territoires, ou lorsque des innovations particulièrement sélectives apparaissent, les mutations locales (villes-champignons, crises, disparitions..) peuvent modifier sensiblement la position des villes dans le système. La 
modélisation de ces interactions non linéaires doit faire appel à une dynamique plus complexe que celle invoquée par Gibrat.

L'expansion des systèmes de villes n'apparaît pas comme un processus continu dans le temps. Longtemps, la vitesse très lente des communications a limité la portée des interactions urbaines et a empêché que les villes ne se libèrent des limites assignées à leur développement par les ressources des territoires accessibles, en général assez peu étendus. Les historiens expliquent ainsi que la proportion de population urbaine se soit maintenue en moyenne, dans les économies pré-industrielles même les plus prospères, aux environs de $10 \%$ (Bairoch 1996). Le processus d'urbanisation, amorcé depuis l'émergence des villes, quelque 2 à 3000 ans après l'apparition de l'agriculture (Bairoch 1985), a connu une accélération considérable lorsque la révolution des transports a permis que s'étendent les territoires et les réseaux d'action dominés par les villes. La transition urbaine, qui a élevé les taux d'urbanisation jusqu'à $80 \%$ dans les pays industriels, en un peu plus d'une centaine d'années, pourrait être ainsi interprétée comme la condensation relativement brutale d'une dynamique de concurrence entre les villes, amorcée bien auparavant, mais bridée par des communications lentes. La transformation des économies agraires en économies industrielles puis tertiaires s'est ainsi accompagnée de modifications drastiques de la répartition du peuplement, conduisant à une trame de villes assez régulièrement espacées, considérablement plus hiérarchisée, diversifiée et concentrée que le système de peuplement antérieur (exemple de la basse vallée du Rhône figure 1).

Pour illustrer la relation entre la forme des systèmes de villes et la vitesse des communications, on peut montrer par exemple que la structure spatiale des systèmes de villes, ainsi que leur degré de hiérarchisation, présente des variantes qui dépendent des modalités de circulation en vigueur au moment où ils se sont mis en place. Ainsi, la longueur de l'espacement moyen entre les villes et l'intensité de leurs contrastes de dimension dans les territoires nationaux ne divisent pas le monde entre pays développés et pays du Tiers-Monde, mais bien entre pays de l'ancien monde et pays de peuplement plus récent (Moriconi-Ebrard 1993). À titre d'exemple, l'espacement moyen entre deux villes de plus de 10000 habitants est, en Chine ou en Europe, inférieur à $15 \mathrm{~km}$, tandis qu'en Amérique du Nord il est de près de $50 \mathrm{~km}$. De même, un indice qui mesure l'importance des contrastes hiérarchiques entre les populations de ces villes, la pente de la distribution «rang-taille», est inférieur à 1 en Europe, et dépasse 1,2 en Amérique du Nord, alors qu'il prend la même valeur en moyenne $(1,05)$ dans les pays développés ou en développement.
En outre, dans tous ces systèmes, on note sur le temps long une tendance à l'accentuation des contrastes hiérarchiques. Celle-ci pourrait s'expliquer par l'avantage initial capté de façon répétée par les grandes villes, du fait de la diffusion hiérarchique des innovations (renforcement de la hiérarchie par le haut). Mais il résulte aussi de façon systématique de la contraction de l'espace-temps, qui permet aux villes plus grandes de court-circuiter la clientèle des villes plus petites en position intermédiaire, induisant de plus une «simplification par le bas» de la hiérarchie urbaine. L'écart le plus souvent observé par rapport au modèle de Gibrat, à savoir une corrélation entre la croissance et la taille des villes, se trouve ainsi incorporé à une théorie évolutive des villes qui les définit comme articulant deux types d'espace relationnels situés à deux échelles de temps différentes et qui prend en compte la tendance historique, soutenue par le progrès technologique, à la contraction de l'espace géographique, lorsqu'il est confronté aux temps de parcours.

La construction progressive d'un système de peuplement de plus en plus hiérarchisé, avec des contrastes de plus en plus grands entre les dimensions de ses éléments, est donc une conséquence, non pas de l'organisation optimale des circulations dans un territoire à un moment donné (ce qui condamne toutes les théories explicatives statiques comme celle des lieux centraux ou de l'équilibre économique spatial), mais bien de l'adaptation progressive, historique, d'un système initialement bâti en fonction d'une certaine vitesse de circulation et qui s'est transformé sous l'effet de vitesses plus rapides. Ce faisant, le système a aussi permis que les sociétés se libèrent progressivement de la dépendance écologique locale, à l'égard des ressources offertes par leur site, et que soient réduits les aléas, les incertitudes menaçant leur survie et leur développement, en élargissant considérablement les possibilités de substitution et de complémentarité de ressources, par l'appel à celles de sites de plus en plus éloignés.

Cette conception permet de résoudre en un concept unifié la dualité organisationnelle entre l'organisation hiérarchique des systèmes de villes assurant le contrôle et la desserte du territoire d'une part, et leur mise en réseau pour l'exercice de fonctions spécialisées variées d'autre part. On n'est plus obligé de concevoir, comme dans la théorie des lieux centraux, qu'il existe a priori des fonctions différenciées par leur seuil d'apparition ou leur portée, qui vont hiérarchiser les villes en dimension et en espacements. Ces fonctions se créent dans les villes, elles émergent du fait d'un processus de division sociale et spatiale du travail qui produit une complexité croissante de niveaux fonctionnels en transformant les lieux pré-existants. 
À quel niveau géographique faut-il construire la théorie et les modèles qui permettent de la tester? On a ici surtout raisonné au niveau agrégé des villes, mais la théorie doit aussi considérer le niveau «microgéographique», celui des acteurs individuels ou collectifs qui composent le milieu social d'une ville, qui sont connectés, informés mutuellement de leurs actions, et qui sont en concurrence avec ceux des autres villes pour capter les profits associés à l'exploitation des innovations. Les routes et les infrastructures qui unissent les villes d'un même système sont en fait des réseaux sociaux par lesquels transitent les informations. Certains de ces réseaux sont organisés hiérarchiquement, de droit (l'administration), ou de fait (la distribution), d'autres non, et la possibilité de leur captage par une ville quelconque du système n'est pas exclue a priori. Une spécialisation réussie à un moment donné résulte de l'exploitation de l'avantage initial conféré par l'adoption précoce d'une innovation économique, sociale ou politique appelée à se développer. L'information concernant la structure du réseau urbain (forme hiérarchique et spécialisations) est connue des acteurs, qui peuvent l'utiliser pour s'y conformer ou pour la contester. En dépit de la diversité de la personnalité des acteurs, de leurs motivations et

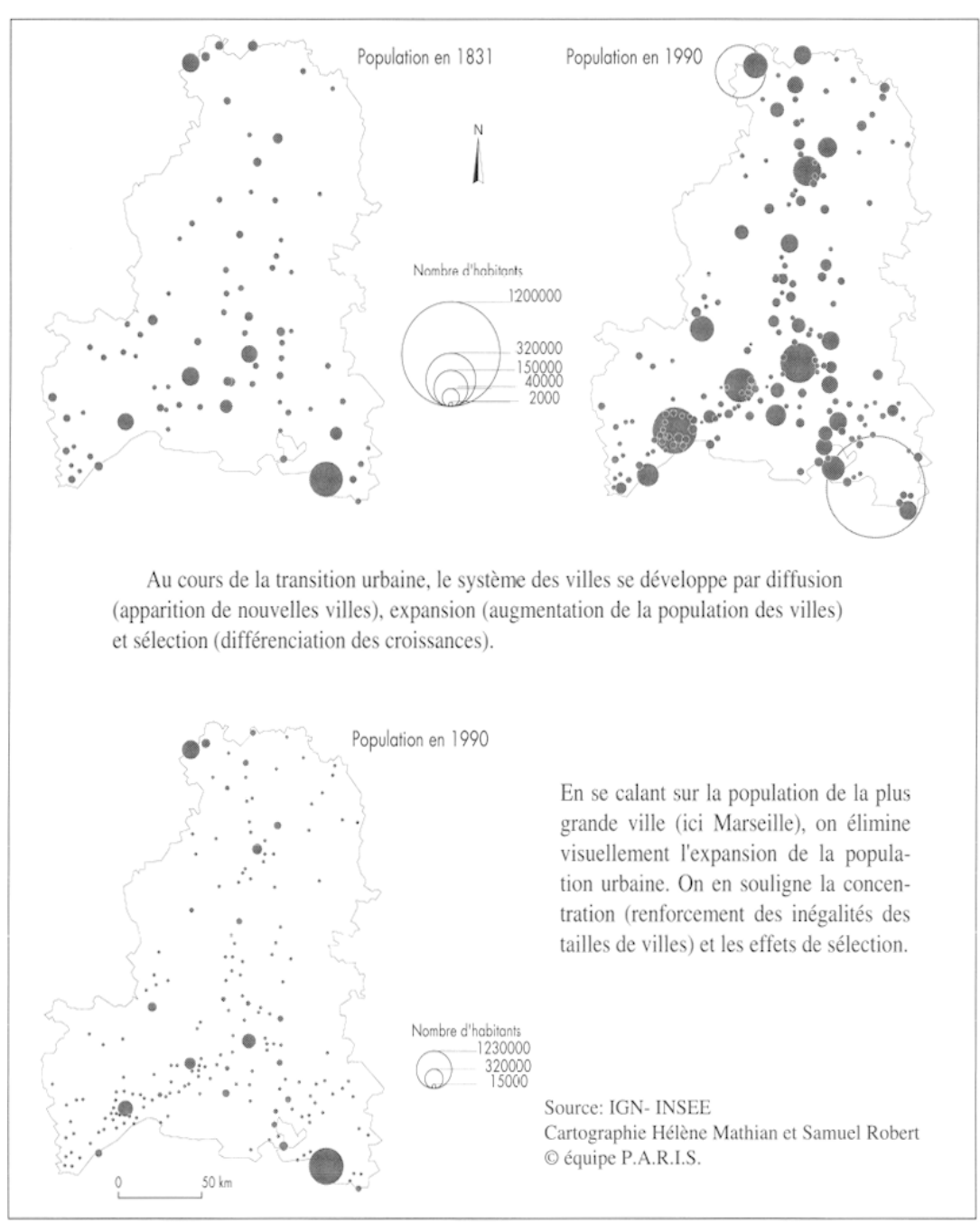

Fig. 1._Processus de développement d'un système de villes. de leurs actions, l'agrégation de leur comportement produit des régularités, souvent indépendantes des finalités qu'ils se sont assignées, et qui peuvent être analysées au niveau de l'évolution d'une ville comme de celle du système des villes, assorties d'un facteur «aléatoire», d'un degré d'imprévisibilité d'importance variable qui correspond à l'ignorance dans laquelle on se trouve des décisions prises par les acteurs.

Les recherches manquent encore pour articuler la forme prise par les réseaux sociaux locaux (dans une ville) à celle de l'ensemble des villes. En particulier, on ne sait pas bien ce qui fait l'efficacité d'une "synergie» locale, d'une forme particulière des relations entre les acteurs dans une ville, qui selon certains faciliterait son adaptation au changement. On identifie assez mal ce qui constitue des niveaux de complexité différents entre des fonctions inégalement spécialisées et adaptables, et plus généralement entre des villes de taille différente. La théorie systémique laisse deviner (le tout

est plus que la somme des parties) mais n'explique pas pourquoi le niveau fonctionnel d'une ville ayant grandi seule régulièrement est plus élevé, d'une plus grande complexité, que celui d'un ensemble de deux ou plusieurs villes agrégeant le même nombre d'habitants ou d'entreprises mais réunies spatialement depuis peu de temps seulement. $\mathrm{Par}$ exemple, la région urbaine de la Ruhr, bien que forte d'une agglomération de population de plus de 10 millions d'habitants, non seulement arrive bien loin derrière Londres et Paris, mais se classe aussi probablement après Madrid ou Milan, en termes de niveau fonctionnel. Tout se passe comme si, alors que les réseaux sociaux se renouvellent par migration des personnes et d'une génération à l'autre, certains savoir-faire urbains se perpétuaient dans les mêmes lieux, ce qui ne peut se comprendre que par un ensemble d'effets en retour et de contraintes exercées par le lieu sur les acteurs, et par des processus d'apprentissage exigeant un 
temps assez long pour apporter des résultats significatifs dans la compétition interurbaine.

Une théorie évolutive prend donc nécessairement en compte ce que l'on appelle la complexité. Est-elle encore traduisible en modèles, statistiques ou mathématiques, qui permettraient de la mettre à l'épreuve? Formaliser les modalités de l'évolution des villes suppose de combiner un processus de croissance systémique à la Gibrat, une concurrence entre les centres pour la captation des ressources et des innovations selon des portées spatiales de plus en plus étendues, une complexification croissante des activités et de la société urbaines selon des tendances historiques spécifiques, qui sont très difficiles à formaliser par les méthodes classiques de modélisation, comme les modèles dynamiques utilisant des équations différentielles. Des méthodes de simulation informatique souple comme celle des systèmes multi-agents, semblent seules à même de réunir dans un seul modèle le nombre de propositions (règles) théoriques élémentaires qui sont nécessaires pour une description même très sommaire, "parcimonieuse », de l'évolution d'un système de villes (Bura et al. 1996).

\section{Conclusion}

Retrouvant le lien essentiel établi par J. Remy et L. Voyé entre ville et communication, puisqu'ils définissent l'urbanisation comme «un processus intégrant la mobilité spatiale à la vie quotidienne» (1992, p.7), nous avons défini la finalité essentielle d'un système urbain dans un territoire comme étant d'articuler deux modes de relation des sociétés à leur environnement, ou encore deux modes de production d'espaces géographiques, ayant chacun leur vitesse de circulation et donc leur échelle d'organisation géögraphique spécifique. Plus que pour d'autres systèmes sociaux, on ne saurait rendre compte des similitudes dans l'organisation spécifique des systèmes de villes, de leur structure universelle dans des milieux géographiques, des systèmes économiques et des organisations sociopolitiques différentes, sans invoquer d'une part cette finalité territoriale commune, et sans d'autre part la mettre en relation avec d'autres processus, transculturels mais néanmoins historiques, que sont la transition urbaine et la contraction de l'espace-temps. La structure actuelle des systèmes de villes est le produit de ces deux processus, qui ont profondément transformé les modalités pratiques de leur fonction principale, de leur signification de l'ordre de l'ingénierie spatiale, qui elle resterait invariante, et qui est d'articuler territoires du quotidien et réseaux des pouvoirs. On peut donc considérer qu'en définitive, la finalité des systèmes urbains consiste à réduire l'aléa local par la mise en connexité des lieux, en réalisant cette adaptation continue par le changement de dimension des deux types de territoire.

Pour dépasser les apories des théories urbaines statiques, on propose donc de concevoir le système de villes comme une forme d'adaptation au changement engendré par les sociétés humaines, une invention dont la technicité n'est en général guère perçue dans les représentations collectives, et qui résulte de processus historiques d'auto-organisation. La spécificité évolutive des systèmes de villes est à rechercher dans au moins trois processus conjoints: dans la transition urbaine, qui fait dériver la quasi-totalité des systèmes de villes par expansion, concentration et diversification d'un semis de peuplement ayant pour fonction originelle l'exploitation agricole d'un territoire; dans le caractère connecté, mutuellement informé et concurrent du réseau que forment d'emblée les établissements humains dès que s'accroissent les densités ou dès qu'apparaissent les villes (la prédation, le commerce, les échanges); et enfin dans le fait que ce réseau est un objet spatial et historique, qui s'enracine dans un territoire ne conservant pas anthropologiquement les mêmes propriétés spatiales au cours du temps. La variation systématique de la portée des relations urbaines, la contraction de l'espace-temps, est un processus essentiel, qui différencie la dynamique des villes de celle d'autres systèmes dynamiques, et qui restitue à la théorie des systèmes de villes sa dimension spécifiquement géographique. La conception évolutive implique aussi que soit prise en compte la capacité créative des centres urbains, le flux des innovations et la création de richesses nouvelles étant seuls à même d'expliquer la poursuite de l'urbanisation.

Une conception évolutive oblige à réviser fondamentalement nos représentations et nos méthodes d'analyse du changement dans l'espace géographique. Un grand régulateur de la dimension des entités territoriales, peut-être le principal, est la portée des interactions spatiales. Il faut la mesurer de façon socialement significative, c'est-à-dire en temps, et non plus en kilomètres. C'est cette mesure de l'espace-temps qui va définir des entités géographiques, non plus fixes, figées par des bornes topographiques ou des partitions administratives, mais évolutives, en expansion dans l'espace-support. Si l'on veut construire une théorie évolutive de ces objets, il faut considérer à des dates différentes le même objet dans une délimitation géographique qui corresponde à la portée de ses interactions du moment. Donnons-en deux exemples: si l'on appelle ville une portion d'espace-support dans laquelle les relations sont polarisées par un centre, auquel il est possible d'accéder en moins d'une heure, alors la même ville va normalement couvrir en 1950 une superficie à peu près double de celle de 1800 , 
et en 1990 environ le double de celle de 1950. Il est probable que sur un tel objet, identifié comme «ville» de manière cohérente dans l'espace-temps relationnel, l'on n'observe plus les alternances de croissance puis de décroissance dans une partie centrale, et de croissance de périphéries de plus en plus éloignées (physiquement) qui ont fait la joie des tenants de la «contre-urbanisation» et qui sont bien décrites par les spécialistes de la périurbanisation. Au contraire, il y a beaucoup de chances pour qu'on découvre que cette ville définie de façon évolutive garde, par opposition à sa périphérie, une tendance continue à la croissance. De même, bien des débats relatifs aux «replis» sur des espaces locaux plus restreints pourraient tout aussi bien être interprétés, à la lumière de l'extension de la portée spatiale des interactions, comme des extensions: ainsi, le «pays», plutôt qu'une restriction du cadre régional, serait l'équivalent du village ou de la commune d'autrefois en termes de possibilités d'interactions, tout comme certaines «fragmentations» correspondent peut-être à l'établissement d'interactions à des échelons jusque-là ignorés par les processus d'intégration spatiale.

Armés de ces notions d'entités spatiales évolutives, les géographes pourraient aussi mieux éclairer certaines questions de prospective: si l'on se demande ce que sera la post-transition urbaine, comment vont évoluer les systèmes de villes, il n'est que d'observer l'accroissement des vitesses de circulation inter-urbaines et l'extension des réseaux de relations pour prédire la poursuite des tendances à la concentration: dans un espace-temps réduit par rapport à l'espace-support, une trame constituée d'un plus petit nombre de villes est l'équivalent transformé de la trame antérieure. Enfin, la perspective évolutive amène à considérer autrement les interrogations relatives à des formes supposées optimales de l'aménagement du territoire: les mêmes mécanismes de changement ont pu produire des formes légèrement différentes de systèmes de villes (par exemple, le réseau centralisé français par opposition aux réseaux de type rhénan), qui sont autant de variétés également viables et non réductibles l'une à l'autre, susceptibles de s'adapter aux changements en cours sans nécessairement rapprocher leurs structures. Il n'y a pas de système de villes optimal, il n'y a que des systèmes durables.

\section{Références}

Allen P. (1978). «Dynamique des centres urbains». Sciences et Techniques, 50, p. 15-19.

ALLEN P. (1991). "Spatial models of evolutionary systems», in Pumain D., Saint-Julien Th., eds., Spatial analysis and population dynamics. Montrouge: John Libbey-INED, Congresses and Colloquia, 6, p. 147-160.
Allen P., Sanglier M. (1979). «A dynamic model of growth in a central place system». Geographical Analysis, p. 256-272.

ARLINGHAUS S.L. (1985). «Fractals take a central place». Geografiska Annaler, 67B, p. 83-88.

AUERBACH F. (1913). «Das Gesetz der Bevölkerungskonzentration». Petermanns Mitteilungen, 59, 1, p. 74-76.

Auray J.P., Bailly A., Derycke P.H., Huriot J.M., dir., (1994). Encyclopédie d'économie spatiale. Paris: Economica.

BAIROCH P. (1985). De Jericho à Mexico. Paris: Gallimard.

BAIROCH P. (1996). «Cinq millénaires de croissance urbaine», in SACHS I., dir., Quelles villes pour quel développement? Paris: Presses universitaires de France.

BatTy M., Longley P. (1994). Fractal cities. London: Academic Press.

BAUMONT C., HuRIOT J.M. (1996). «La ville et ses représentations formelles». in Derycke P.H., HuRiot J.M., Pumain D., dir., Penser la ville: théories et modèles. Paris: Anthropos, coll. «Villes», p. 7-51.

BeCKMANN M.J., MacPHeRson J.C. (1970). "City size distribution in a central place hierarchy». Joumal of Regional Science, 10, 1, p. 25-33.

BEGUIN H. (1979). «Urban hierarchy and the rank-size distribution». Geographical Analysis, 11, 2, p. 149-163.

Beguin H. (1988). «La région et les lieux centraux», in PonSARD C., dir., Analyse économique spatiale. Paris: Presses universitaires de France.

BEGUIN H. (1992a). «Christaller's central place postulates: a commentary». The Annals of Regional Science, 26, p. 209-229.

BEGUIN H. (1992b). "Postulates in Lösch's theory of economic regions». Sistemi Urbani, 14, p. 45-59.

BEguin H. (1996). «Faut-il définir la ville?» in DeRYCKE P.H. Huriot J.M., Pumain D., dir., Penser la ville: théories et modèles. Paris: Anthropos, coll. «Villes», $337 \mathrm{p}$.

BEGUIN H., THISSE J.-F. (1979). «An axiomatic approach to geographical space». Geographical Analysis, 11, 325-341.

Berry B.J.L. (1964). «Cities as systems within systems of cities». Papers of the Regional Science Association.

BERRY B.J.L. (1967). Geography of Market Centers and Retail Distribution. New York, trad. franç. par B. Marchand, Paris: PUF, 1973.

BONITZER J. (1984). Psychologie du hasard. Paris: Messidor.

Bura S., Guérin-Pace F., Mathian H., Pumain D., Sanders L. (1996). «Multi-agents systems and the dynamics of settlement systems». Geographical Analysis, p. 161-178.

CAMAGNI R. (1993). Economia urbana. Milan: Franco Angeli.

CARROLL G.R. (1982). «National City-size distributions: what do we know after 67 years of research?» Progress in Human Geography, 6, 1.

CATIN M. (1994). «Les économies d'agglomération», in AURAY J.P., Bailly A., Derycke P.H., Huriot J.M., dir., Encyclopédie d'économie spatiale. Paris: Economica.

Cauvin C., Reymond H. (1985). L'Espacement des villes, Paris: CNRS, coll. «Mémoires et Documents de Géographie».

Cauvin C., Reymond H., Schaub R. (1989). «Accessibilité, temps de séjour et hiérarchie urbaine». Sistemi Urbani, 3, p. 297-324.

Chevalier M. (1832). «Système de la Méditerranée». Le Globe, 12 février.

Christaller W. (1933). Die zentralen Örte in Süddeutschland : eine ökonomisch-geographische Untersuchung über die gesetz Massigkeit der Verbreitung und Entwicklung der Siedlungen mit städitschen Funktionen. Jena: Fischer Verlag,(trad. anglaise : Central places in Southern Germany. Englewood Cliffs, N.J.: Prentice Hall).

ClaVal P. (1982). La Logique des villes. Paris: LITEC. 
CURRY L. (1964). «The random spatial economy: an exploration in settlement theory». Annals of the Association of American Geographers, p. 138-146.

DendRINOS D., Mullaly H. (1985). Urban evolution. Oxford: Oxford University Press.

DERYCKE P.H. (1996). «Équilibre spatial urbain», in DERYCKE P.H., Huriot J.M., Pumain D., dir., Penser la ville: théories et modèles, Paris: Anthropos, p. 53-90.

DupuY G. (1995). Les Territoires de l'automobile. Paris: Anthropos, coll. «Villes».

FLETSCHER R. (1986). "Settlement in Archaeology : world-wide comparison». World Archaeology, 18, 1, p. 59-83.

François N., Frankhauser P., Pumain D. (1995). «Villes, densité et fractalité». Annales de la Recherche urbaine, 67, 54-63.

FrankHAUSER P. (1993). La Fractalité des structures urbaines. Paris: Anthropos.

FuJiTA M. (1989). Urban economic theory. Land use and city size. Cambridge : Cambridge University Press.

FuJiTA M., KRUGMAN P., MORI T. (1994). On the evolution of hierarchical urban systems. Niagara Falls, North American Meeting of the Regional Science Association International, $80 \mathrm{p}$.

GiBRAT R. (1931). Les Inégalités économiques. Paris: Sirey.

GRASLAND C. (1994). «Limites politiques et barrières migratoires», in Gallusser W.A., ed., Political boundaries and coexistence. Bâle: Peter Lang, p. 425-440.

GreEnwood M. J. (1981). Migration and Economic Growth in the United States. New York: Academic Press.

GuÉRIN-PACE F. (1993). Deux siècles de croissance urbaine. Paris: Anthropos.

HAKEN H. (1977). Synergetics, an introduction. Berlin: Springer.

JUILLARD É. (1972). "Espace et temps dans l'évolution des cadres régionaux", in Études de géographie tropicale offertes à P. Gourou. Paris: Mouton, p. 29-43.

LAHERRÈre J. (1995). «Distributions de type "fractal parabolique" dans la Nature». C.r. de l'Académie des Sciences. Paris, t. 322, IIa, p. 535-541.

Le Bras H. (1996). Le Peuplement de l'Europe. Paris: La Documentation Française.

LePETIT B., PuMAin D. (dir.) (1993). Temporalités urbaines. Paris: Anthropos.

Marchetti C. (1986). Some Phenomenological Observations on Urban Dynamics. Laxenburg: IIASA.

Moriconi-EBRARD F. (1993). L'Urbanisation du monde depuis 1950. Paris: Anthropos.

ORFEUIL J.P. (1993). Je suis l'automobile. Paris: Éditions de l'Aube.

PhILBRICK A.K. (1957) "Principles of areal functioning of organization in regional human geography ». Economic Geography, 33, p. 299-336.

PRED A. (1966). The spatial dynamics of US industrial growth 1800 1914. Cambridge, Mass.: MIT Press.

PRED A. (1973). «Systems of cities and information flows». Lund Studies in Geography, Serie B, 38.

PRED A. (1977). City systems in advanced societies. Londres: Hutchison.

Pumain D. (1980). Contribution à l'étude de la croissance urbaine dans le système urbain français. Paris: Université Paris I, thèse de doctorat.

Pumain D. (1982). La Dynamique des villes. Paris: Economica.

Pumain D. (1994). «Villes et agglomérations urbaines », IN AURAY
J.P., Bailly A., DeRYcke P.H., HuRIOT J.M., dir.: Encyclopédie d'Économie spatiale. Paris: Economica, chap. 15, p. 111-125.

PUMAIN D. (1995a). «Implicit large territorial-technical system: settlement systems». Flux, 21, p. 11-20.

Pumain D. (1995b). «Le peuplement», in Bailly A., Ferras R., Pumain D., dir., Encyclopédie de géographie. Paris: Economica, $2^{\mathrm{e}}$ éd.

PuMAIN D., HAAG G. (1994). «Spatial patterns of urban systems and multifractality», in Evolution of Natural Structures, 3rd International Symposium of the Sonder-forschungsbereich 230, Universität Stuttgart, 4-7 oct., Natürliche Konstruktionen, Mitteilungen des SFB 230, Heft 9, p. 243-252.

PUMAIN D., MORICONI-EBRARD F. (1997). «City size distributions and metropolisation», Geojournal.

Pumain D., Robic M.-C. (1996). «Théoriser la ville», in DerYcke P.H., Huriot J.M. Pumain D., dir., Penser la ville: théories et modèles. Paris: Anthropos, p. 107-161.

Pumain D., SAINT-Julien Th. (1978). Les Dimensions du changement urbain. Paris: CNRS.

Pumain D., SAINT-JuliEN Th. (1989). «Migrations et changement urbain». Revue d'économie régionale et urbaine, 3, p. 509-530.

Pumain D., Sanders L., Saint-Julien Th. (1989). Villes et autoorganisation. Paris: Economica.

ReCLuS É. (1895). «The evolution of cities». The Contemporary Review, 67, 2, p. 246-264.

REINER T.A., PARR J.B. (1980). «A note on the dimensions of a national settlement pattern». Urban Studies, 17, p. 223-230.

Remy J., Voyé L. (1992). La Ville: une nouvelle définition. Paris: L'Harmattan.

REYMOND H. (1981). «Une problématique théorique», in ISNARD H., RaCine J.B., Reymond H., Problématiques de la géographie. Paris: Presses universitaires de France.

REYNAUD J. (184). "Villes», in Encyclopédie nouvelle. Paris: Gosselin, t. VIII, p. 670-687 (cité par Robic 1982).

RobIC M.-C. (1982). «Cent ans avant Christaller, une théorie des lieux centraux». L'Espace géographique, 1, p. 5-12.

Robic M.-C. (1984). «Jean Reynaud (1806-1863)», in PINCHEMEL P., RoBIC M.-C., TISSIER J.-L, Deux siècles de géographie française. Choix de textes. Paris: Comité des Travaux historiques et scientifiques, 34-35.

RoBSON B.T. (1973). Urban growth, an approach. Londres: Methuen.

RousSEAU M.P., Prudhomme R. (1993). Les Bénéfices de la concentration parisienne. Paris: IAURIF, $40 \mathrm{p}$.

SANDERS L. (1992). Système de villes et synergétique. Paris: Anthropos.

SANDERS L. (1993). «Modèles de la dynamique urbaine, une présentation critique», in LEPETIT B., PUMAIN D., dir., Temporalités urbaines. Paris: Anthropos, p. 3-41.

VRIES J. DE (1984). European Urbanization 1500-1800. Londres: Methuen.

Weidlich W., HaAG G. (1988). Interregional Migration. Berlin: Springer Verlag.

WhITE R.W. (1977). «Dynamic central place theory». Geographical Analysis, 10, p. 226-243.

WILSON A. (1981). Catastrophe theory and bifurcation. Londres: Croom Helm.

ZIPF G.K. (1949). Human Behaviour and the Principle of Least Effort. Cambridge: Addison Wesley. 